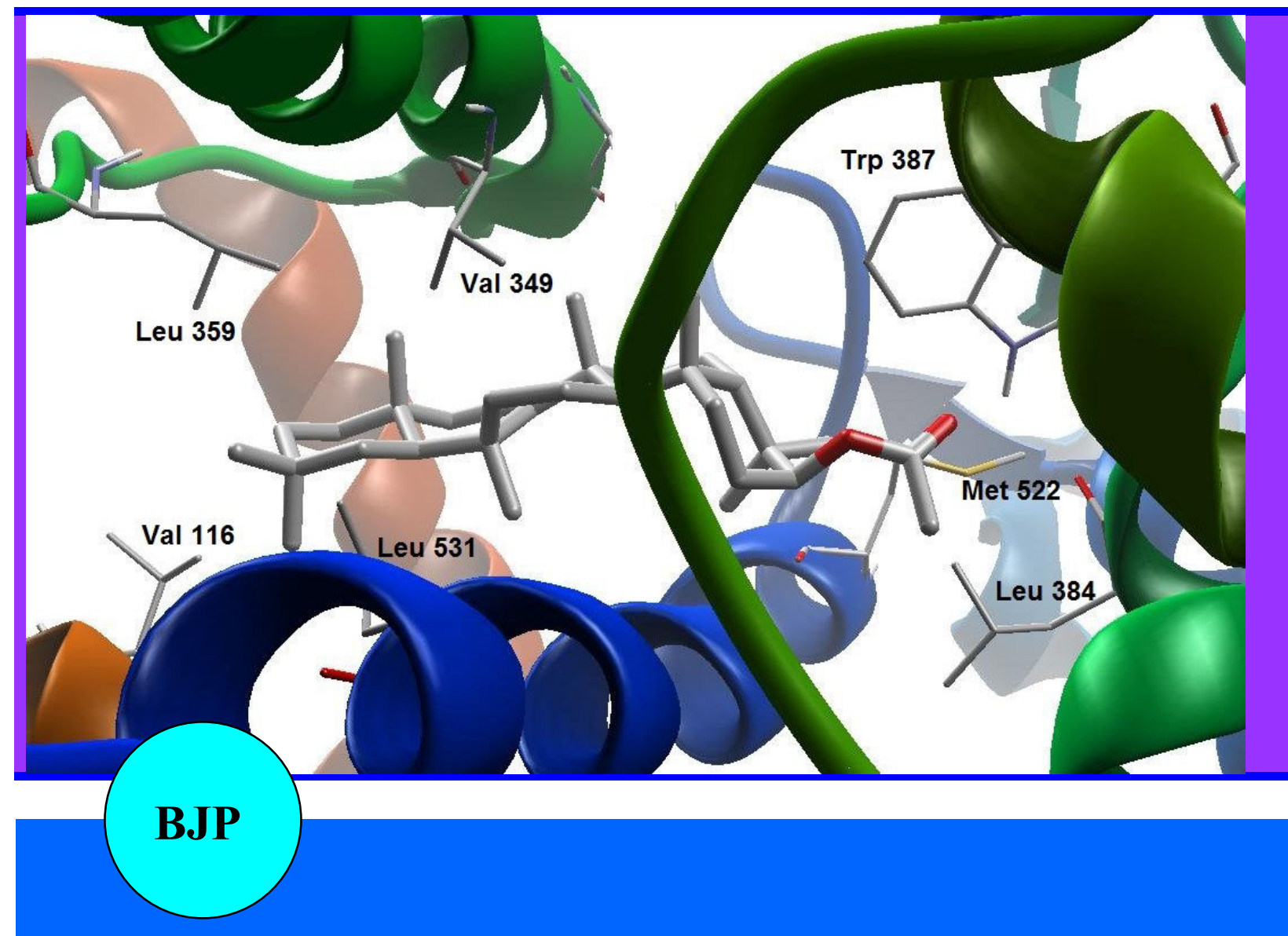

Bangladesh Journal of Pharmacology

Research Article

Molecular docking of taraxerol acetate as a new COX inhibitor 
Abstracted/indexed in Academic Search Complete, Agroforestry Abstracts, Asia Journals Online, Bangladesh Journals Online, Biological Abstracts, BIOSIS Previews, CAB Abstracts, Current Abstracts, Directory of Open Access Journals, EMBASE/Excerpta Medica, Google Scholar, HINARI (WHO), International Pharmaceutical Abstracts, Open J-gate, Science Citation Index Expanded, SCOPUS and Social Sciences Citation Index

ISSN: $1991-0088$

\title{
Molecular docking of taraxerol acetate as a new COX inhibitor
}

\author{
Ubaid Ur Rehman',2, Jasmin Shah', Mudasser Ahmed Khan', Muhammad Raza Shah3, \\ Ishtiaq ${ }^{4}$ and Inamullah Khan 5 \\ ${ }^{1}$ Institute of Chemical Sciences, University of Peshawar, Peshawar 25120, Pakistan; ${ }^{2}$ Department of Biochemistry, \\ Khyber Medical College Peshawar. 25120, Pakistan; ${ }^{3} \mathrm{HEJ}$ Research Institute of Chemistry, International Center for \\ Chemical and Biological Sciences, University of Karachi, Karachi 75270, Pakistan; ${ }^{4}$ Department of Chemistry, \\ Hazara University, Mansehra, Pakistan; ${ }^{5}$ Department of Pharmacy, University of Peshawar, Peshawar 25120, \\ Pakistan.
}

\begin{tabular}{|lr|}
\hline Article Info & \\
\hline Received: & 16 March 2013 \\
Accepted: & 22 March 2013 \\
Available Online: & 2 May 2013 \\
DOI: 10.3329/bjp.v8i2.14167 & \\
& \\
Cite this article: & \\
Rehman UU, Shah J, Khan MA, Shah \\
MR, Ishtiaq, Khan I. Molecular dock- \\
ing of taraxerol acetate as a new COX \\
inhibitor. Bangladesh J Pharmacol. \\
2013; 8: 194-97.
\end{tabular}

\begin{abstract}
Cycloxygenase inhibitors are one of the main class of therapeutic agents for management of inflammation. New COX inhibitors are discovered from natural and synthetic sources. In the current investigation taraxerol acetate have been discovered as a new COX inhibitor. Taraxerol acetate showed considerable inhibitory activity against both COX-1 ( $\left.\mathrm{IC}_{50}: 116.3 \pm 0.03 \mu \mathrm{M}\right)$ and COX-2 ( $\left.\mathrm{IC}_{50}: 94.7 \pm 0.02 \mu \mathrm{M}\right)$ enzymes using in vitro enzyme inhibition assay. Molecular docking revealed significant interactions of taraxerol acetate with the important amino acid residues surrounding the inhibitor in binding pocket of COX-2 enzyme. This study indicate potential of taraxerol acetate to be further explored and modified as a new lead compound for better management of inflammatory conditions via targeting COX enzymes.
\end{abstract}

\section{Introduction}

Cycloxygenases (COX) are involved in biosynthesis of prostaglandins (PGs) as major cytokines playing critical role in inflammations. Compounds with promising potential of COX inhibition can show therapeutic effects in conditions associated with inflammations (Khan et al., 2011). Keeping the background in view, a phytochemical study was conducted to isolate bioactive compounds from Artemisia roxburghiana Besser, with potential therapeutic effect in diseases including inflammation. A. roxburghiana is a perennial aromatic herb belonging to the family Asteraceae. In Pakistan, where the plant is native and occupying dry open slopes and waste lands of the Himalayan region, various ethnobotanical uses have been reported. These include for treating fever, rheumatism, malaria, dysentery and hepatitis (Dua et al., 2011; Ashraf et al., 2010). The aerial parts are also used to treat diabetes either alone or in combination with other plants such as the dried fruits of Zizyphus jujube (Ashraf et al., 2010). In support of this latter traditional use, the ethanolic extract of $A$. roxburghiana from Pakistan has previously shown to display insulin secretagogue activity in cultured insulinoma cell line, INS-1 cells (Hussain et al., 2004). Apart from preliminary phytochemical studies such as essential oils analysis by GC-MS (Ashraf et al., 2010; Bicchi et al., 1998). In case of potential active constituents of $A$. roxburghiana against inflammation no work. In the current investigation we have made a successful effort to identify taraxerol acetate, being the potential chemical constituent, responsible for its reported ethnomedicinal use and pharmacological activity as an anti-inflammatory agent that at least partially targets COX.

\section{Materials and Methods}

\section{Plant material}

The aerial parts (green stems with leaves) of $A$. roxburghiana were collected from the University campus of Hazara University Mansehra, Pakistan. The authentica- 
ted voucher specimen (no 3486) was then deposited at the Herbarium of Hazara University, Mansehra Pakistan. The plant materials were shade dried and ground into powder.

\section{Extraction and isolation}

The powdered plant materials $(17 \mathrm{~kg})$ were extracted 3 times by soaking with $24 \mathrm{~L}$ of methanol for two weeks. Combine extract were filtered and evaporated under reduced pressure to yield $1.5 \mathrm{~kg}$ of the crude extract. Preliminary fractionation of the crude extract was done by suspending the crude extract in water and extraction using solvents of increasing polarity: Hexane, chloroform, ethyl acetate and $n$-butanol. Remaining unfractionated crude extract was taken as aqueous fraction. The ethyl acetate fraction (160 g) that showed the highest PTP1B inhibitory activity was subjected to silica gel column chromatography with hexane containing increasing percentage of ethyl acetate used as eluents. Fractions obtained from the 4.0:6.0 to 0:10

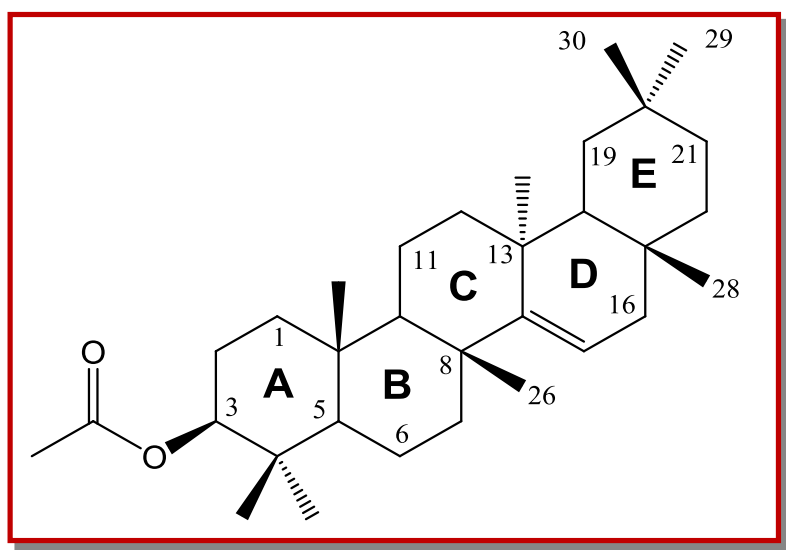

Figure 1: Chemical structure of taraxerol acetate

hexane:EtOAc mixtures were further subjected to CC under the same conditions to yield taraxerol acetate $\left(1.71 \mathrm{~g}, \mathrm{R}_{\mathrm{F}} 0.75\right.$ in hexane:ethyl acetate 0.5:9.5; Figure 1). The structure of isolated compound was confirmed by comparing their corresponding NMR and mass spectral data with those previously reported (Ayatollahi et al., 2011).

\section{Cyclooxygenase (COX) inhibition assay}

Tris buffer ( $50 \mathrm{mM}$; pH 7.5) was used to dissolve both the enzymes i.e. COX-1 and COX-2 enzymes (Cayman Chemicals, USA), co-factors adrenaline (5 mM), and hematin $(1 \mu \mathrm{M})$. Before this hematin was dissolved in $\mathrm{NaOH}$. This reaction mixture was poured into 96-well plates while the final volume was, $190 \mu \mathrm{L}$ in each well. Now the same procedure was adopted for the test sample. Different concentrations of taraxerol acetate were diluted in Tris buffer and then put into a 96-well plate, then cofactor and enzyme $(10 \mathrm{U} / \mathrm{mL} ; 180 \mu \mathrm{L})$ was added to this plate. Before the addition of arachidonic acid these plates were incubated at $37^{\circ} \mathrm{C}$ for $30 \mathrm{~min}$, followed by the addition of $10 \mu \mathrm{L}$ of $1 \mathrm{M} \mathrm{HCl}$ to stop the reaction. Then $10 \mu \mathrm{L}$ of $1 \mathrm{M} \mathrm{NaOH}$ was added to adjust the $\mathrm{pH}$ to 7.5. The formation of $\mathrm{PGE}_{2}$, a metabolite of $\mathrm{PGH}_{2}$ was measured and from this the enzymes activity and the inhibitory effect of different concentrations of taraxerol acetate was assessed. Radioimmunoassay technique was used for measurement of $\mathrm{PGE}_{2}$ contents. The data was presented as percentage control $\mathrm{PGE}_{2}$ production. All the experiments were performed in triplicate and EZFit Enzyme Kinetics program (Perrella Scientific Inc., USA) was used to determine the $\mathrm{IC}_{50}$ values of test samples as well as of standard sample, while results were expressed as Mean \pm SEM.

\section{Molecular docking simulations}

Ligand file (taraxerol acetate) was designed and optimized using dreading force field was implemented in Marvin Sketch V5.1. Molecular coordinates were further optimized using MMFF force field. FRED 2.1 (Khan et al., 2011) was used to dock the OMEGA pregenerated multi-conformer library. Default FRED protocol was used except for the size of the box defining the binding sites. In an attempt to optimize the docking-scoring performance, exhaustive docking was performed with shapegauss applying the "Optimization" mode. The "Optimization" mode involves a systematic solid body optimization of the top ranked poses from the exhaustive docking. 3 different boxes were explored for COX-2 (PDB ID: 3PGH). Three different simulations were carried out with an added value of $9 \AA$ around the active site. After completion, best scoring pose was selected to study molecular interactions behind significant enzyme inhibitory activity of taraxerol acetate.

\section{Molecular docking simulations}

Molecular docking simulations were performed using AutoDock 4.2. along with AutoDock tools using Lamarckian genetic algorithm, as reported earlier (Khan et al., 2010). The X-ray coordinates used in the study were based on the inhibition of COX-2 (PDB ID: 3BGP). The standard docking procedure was used for a rigid protein and a flexible ligand whose torsion angles were identified (for 10 independent runs per ligand). A grid of $60 \times 60 \times 60$ points in three dimensions was built, centered on the catalytic pocket of acetylcholinesterase. A grid spacing of $0.375 \AA$ and a distance dependent function of the dielectric constant were used for the calculation of the energetic map. All other parameters were used according to default settings of AutoDock 3.0.5. On successful completion of docking and clustering analysis, pose with the most favorable free energy (binding energy) was selected as a resultant coordinate.

\section{Results and Discussion}

COX-2 is one of the integral enzyme systems, which through biosynthesis of prostaglandins (PGs) initiate 


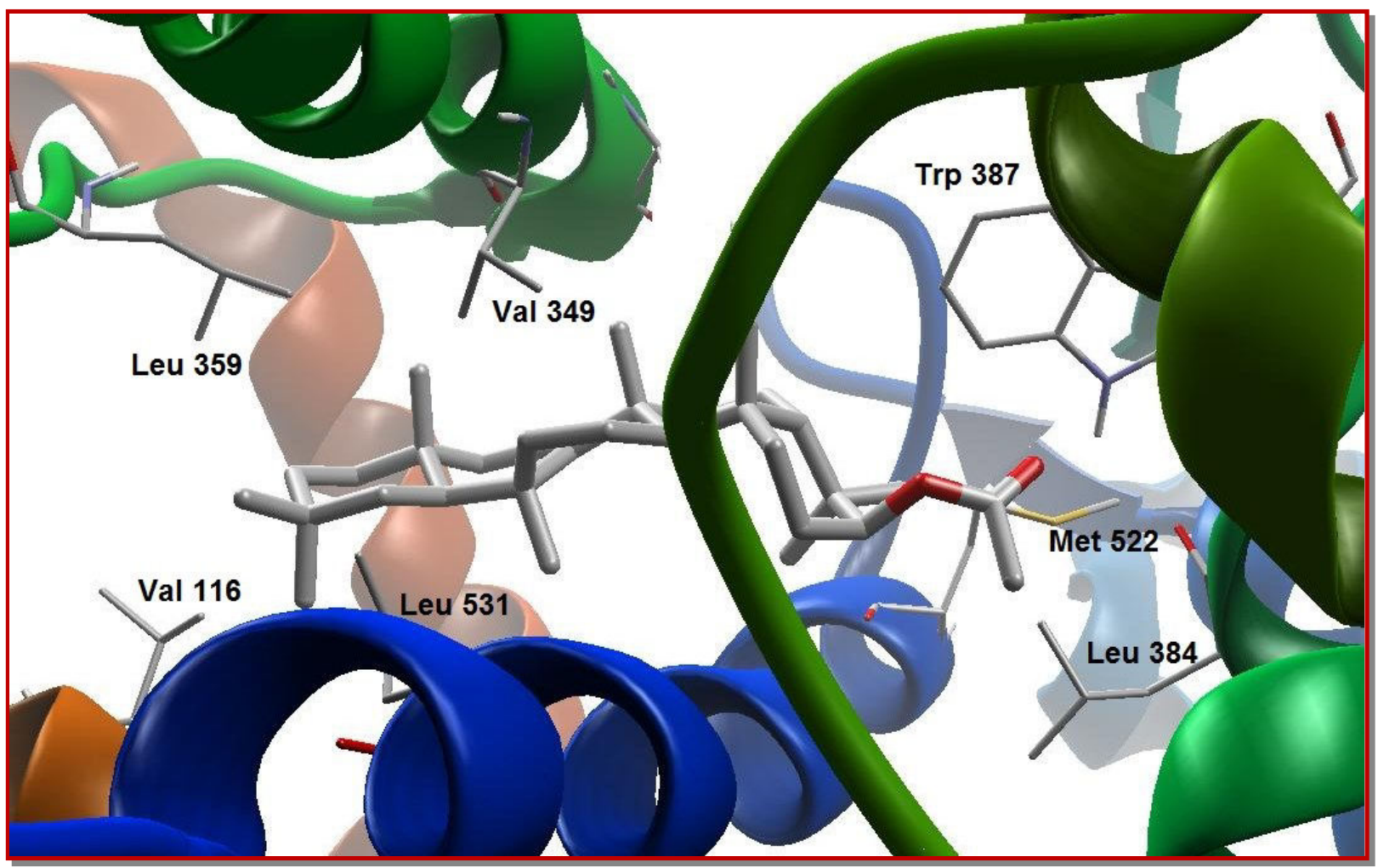

Figure 2: Molecular binding mode of taraxerol acetate inside active site of COX-2

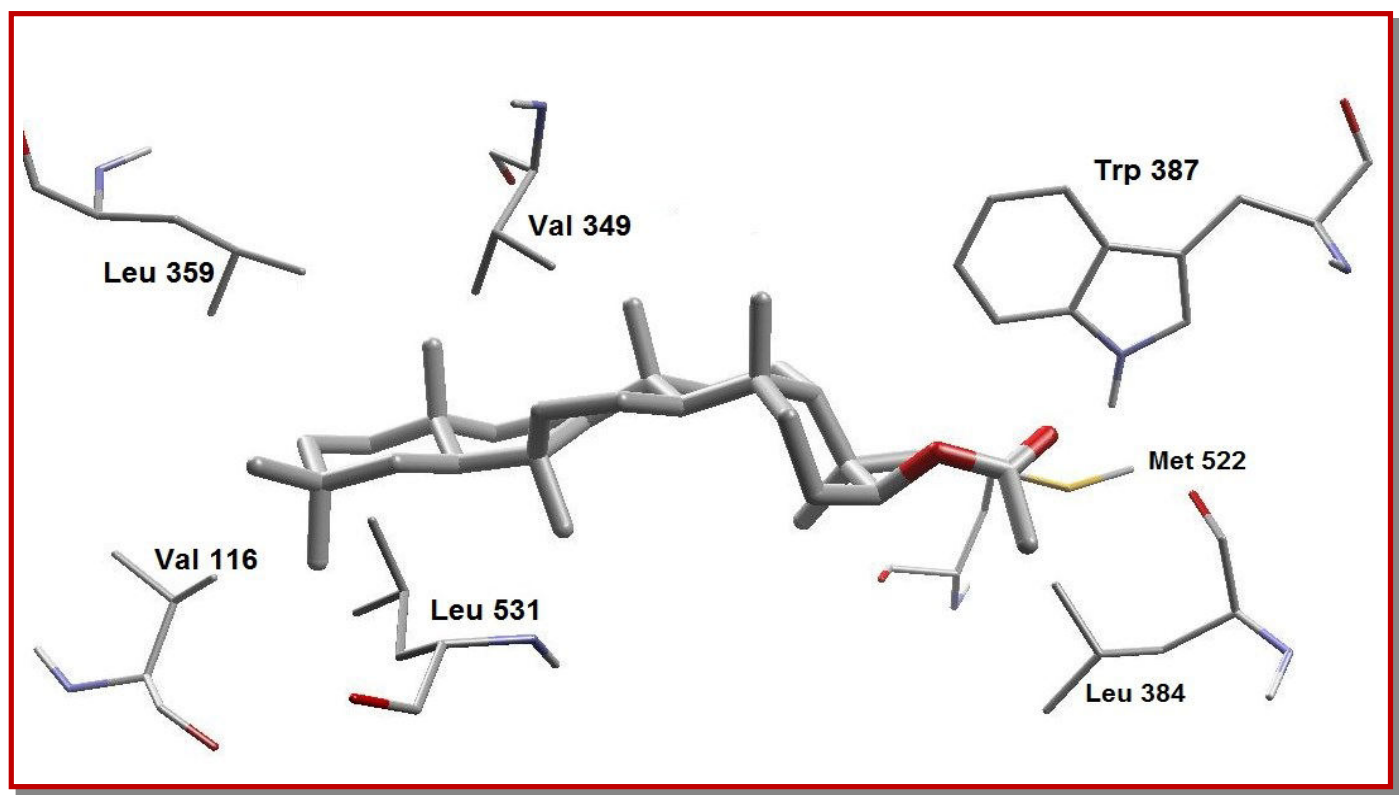

Figure 3: A closer view of taraxerol acetate inside active site of COX-2

inflammatory response. Heme portion is involved in the PGs biosynthesis which is surrounded with in the pocket formed by amino acids residues (Arg120 and Tyr355). These two amino acids have a critical role in designing or virtual screening for discovery of new COX inhibitors. The substrate can only interact with haeme by accessing through the narrow pocket. To validate the accuracy of binding mode and binding affinity flurbiprofen was used as reference and cocrystallized ligand. The binding affinity was determined in terms of binding energy in Kcal/mol. Best scoring conformation of flurbiprofen was found very close to the experimental conformation (RMSD value: 0.453 ). 
After internal validation, molecular docking simulations of the test compound were carried out. The bulky and somewhat less polar surface area of taraxerol acetate made it unfavorable for interaction with the binding pocket of COX-2. However some favorable hydrophobic and electrostatic interactions provided little inhibitory effect against COX-1 ( $\mathrm{IC}_{50}: 116.3 \pm 0.03$ $\mu \mathrm{M})$ and COX-2 $\left(\mathrm{IC}_{50}: 94.7 \pm 0.02 \mu \mathrm{M}\right)$ to the taraxerol acetate inside active site of COX-2, which could be a reason for weak inhibitory effect on direct catalytic activity of COX-2 enzyme. Methyl groups (at positions C-29 and C-30) favorably interact with the less polar amino acid residues Val116 on one side (Figure 2 and 3). On other side, Leu531 also support the ligand inhibitor complex via similar hydrophobic contacts with the same methyl moieties (at positions C-29 and C-30). In addition, Val349 showed hydrophobic interactions with the $\mathrm{C}-11$ and $\mathrm{C}-12$ positions of the ring $\mathrm{C}$ of pentacyclic core of the compound. Leu359 showed favorable hydrophobic interactions with ring $\mathrm{E}$ of the pentacyclic main skeleton of taraxerol acetate. Apart from the main hydrophobic interactions of taraxerol acetate with COX2, acetyl group also revealed its minor hydrophobic contact via its methyl moiety with the methyl groups of Leu384.

Side chain acetate moiety is the only group that provided taraxerol acetate to hold favorable electrostatic interactions with closely surrounding amino acid residues that provided a molecular basis for its weak inhibitory effect on COX-2. Acetyl group showed favorable electrostatic interactions with Trp387 and Met522. Amide group of Leu384 further supported this macromolecular complex via electrostatic interactions with the acetyl group that ultimately led to weak COX-2 inhibitory activity of taraxerol acetate.

\section{References}

Ashraf M, Hayat MQ, Mumtaz AS. A study on elemental contents of medicinally important species of Artemisia L. (Asteraceae) found in Pakistan. J Med Plants Res. 2010; 4: 256-63.

Bicchi C, Rubiolo P, Marschall H, Weyerstahl P, Laurent R. Constituents of Artemisia roxburghiana Besser essential oil. Flavour Fragrance J. 1998; 13: 40-46.

Dua VK, Verma G, Agarwal DD, Kaiser M, Brun R. Antiprotozoal activities of traditional medicinal plants from the Garhwal region of North West Himalaya, India. J Ethnopharmacol. 2011; 136: 123-28.

Khan I, Nisar M, Ebad F, Nadeem S, Saeed M, Khan H, Samiullah, Khuda F, Karim N, Ahmad Z. Anti-inflammatory activities of Sieboldogenin from Smilax china Linn.: Experimental and computational studies. J Ethnopharmacol. 2009; 121: 175-77.

Khan I, Nisar M, Khan N, Saeed M, Nadeem S, Fazal-urRehman, Ali F, Karim N, Kaleem WA, Qayum M, Ahmad $\mathrm{H}$, Khan IA. Structural insights to investigate Conypododiol as a dual cholinesterase inhibitor from Asparagus adscendens. Fitoterapia 2010; 81: 1020-25.

Khan I, Nisar M, Shah MR, Shah H, Gilani SN, Gul F, Abdullah SM, Ismail M, Khan N, Kaleem WA, Qayum M, Khan H, Obaidullah, Samiullah, Ullah M. Anti-inflammatory activities of Taxusabietane A isolated from Taxus wallichiana Zucc. Fitoterapia 201; 82: 1003-07.

Fisher EH, Charbonneau H, Tonks NK. Protein tyrosine phosphatases: A diverse family of intracellular and transmembrane enzymes. Science 1991; 253: 401-06. 